\title{
Aquisição e persistência de memória implícita em adultos saudáveis
}

\author{
Acquisition and persistence of implicit memory in healthy \\ adults
}

\author{
Roberta de Figueiredo Gomes ${ }^{1}$, Martín Pablo Cammarota ${ }^{2}$, Mirna \\ Wetters Portuguez ${ }^{3}$
}

1.Neuropsicóloga, Doutoranda em Medicina e Ciências da Saúde, Neurociências pela Pontifícia Universidade Católica do Rio Grande do Sul, PUCRS. Porto Alegre-RS, Brasil.

2.Biólogo, Doutor, Professor titular do Instituto de Cérebro do Rio Grande do Norte. Natal-RN, Brasil.

3.Neuropsicóloga, Doutora, Professora adjunta da Pontifícia Universidade Católica do Rio Grande do Sul, PUCRS. Porto Alegre-RS, Brasil.

\section{Resumo}

Objetivo. Avaliar a aquisição e persistência da memória implícita e comparar seu desempenho entre dois grupos (grupo 1: 30-40 anos; grupo 2: 60-70 anos), com gênero, escolaridade, classe socioeconômica, sintomas depressivos e ansiosos. Método. Estudo transversal, realizado no ambulatório de terceira idade da unidade de neuropsicologia do hospital São Lucas/PUCRS, academias de ginástica e escolas de idiomas. Os sujeitos responderam a uma entrevista para exclusão de medicação psiquiátrica e doença neurológica. Para estimulação da memória implícita foi utilizado Hooper Visual Organization Test (VOT). Resultados. Houve uma diferença entre os grupos, demonstrando que o grupo 2 apresenta pior desempenho da memória implícita quando comparado ao grupo 1 . Não houve diferença em relação ao gênero, classe socioeconômica, ansiedade, depressão e escolaridade. Conclusão. A avaliação com VOT mostrou que o grupo 1 apresenta um desempenho da memória implícita melhor do que o grupo 2 . Sintomas de ansiedade não interferem em seu desempenho. Não houve diferença entre os gêneros e classe socioeconômica.

Unitermos. Memória implícita; aprendizagem; envelhecimento; ansiedade; depressão

\begin{abstract}
Objective. To evaluate the acquisition and persistence of implicit memory and compare its performance between two groups (group 1: 30-40 years old; group 2: 60-70 years old), regarding gender, schooling, socioeconomic class, depressive, and anxious symptoms. Method. Cross-sectional study, carried out at the senior outpatient Clinic, São Lucas hospital/PUCRS neuropsychology unit, gyms and language schools. The subjects answered to an interview for exclusion of psychiatric medication and neurological disease. For stimulating implicit memory, the Hooper Visual Organization Test (VOT) was used. Results. There was a difference between the two groups, demonstrating that group 2 shows worse performance of implicit memory when compared with group 1. Conclusion. The VOT evaluation showed that group 1 had significantly better performance of implicit memory than group 2. Anxiety symptoms do not interfere with its performance. There was no difference relating to gender, socioeconomic class, anxiety, depression and schooling.
\end{abstract}

Keywords. Implicit memory; learning; aging; anxiety; depression

Trabalho realizado na Pontifícia Universidade Católica do Rio Grande do Sul, PUCRS. Porto Alegre-RS, Brasil. 


\section{INTRODUÇÃO}

Nos últimos anos, o funcionamento cognitivo do cérebro e os processos de consolidação de memória vêm sendo alvo de muitas pesquisas, visando o entendimento das bases biológicas do comportamento. Estudos com animais foram complementados em seres humanos amnésicos e também por estudos de neuroimagem em voluntários normais, mas ainda há poucas evidências dos mecanismos pelos quais o cérebro adquire, armazena e evoca as informações ${ }^{1}$. Ainda, parece claro que algumas tarefas são aprendidas dentro de regiões cerebrais específicas. Esses achados surgiram a partir de estudos com indivíduos que apresentavam lesões cerebrais delimitadas, acompanhadas por déficits de memória.

Os primeiros estudos acerca da aprendizagem se detiveram no hipocampo e do seu papel no aprendizado e na memória. As lesões nessa área parecem impedir o surgimento de memórias utilizadas para aprendizado de novos fatos ou eventos e surpreendentemente, outros tipos de memória permaneceram intactas ${ }^{2}$.

Sabe-se que as memórias sofrem influências fisiológicas e ambientais em seu desempenho conforme a idade. Um dado bem aceito pelos pesquisadores é que a memória consciente (explícita) é mais sensível aos efeitos do envelhecimento do que a memória inconsciente (implícita) ${ }^{3}$.

Em relação ao processo de memorização, este é composto por três estágios consecutivos: aquisição 
(formação do traço base de memória), armazenamento (conservação, alteração ou perda da informação) e evocação de informações (recuperação diante de estímulos internos ou externos), sendo o conjunto das memórias o que se denomina personalidade 4 .

Estudos em modelos animais sugerem que a formação da memória envolve uma série de alterações bioquímicas em diversas áreas do sistema nervoso central, entre as quais se destaca o hipocampo. Em ratos, observam-se essas alterações ou pelo menos algumas delas ocorrendo no hipocampo, amígdala e em diferentes áreas corticais quando os animais são submetidos a um novo aprendizado ${ }^{5}$.

Porém, muitos procedimentos interferem na formação das memórias. Estudos demonstram que para a construção de uma memória de longo prazo são necessárias, nas primeiras três a seis horas, a expressão gênica e a síntese proteica no hipocampo ou em outras regiões cerebrais ${ }^{6}$, correspondendo a modificações permanentes ou muito duradouras na forma e função das sinapses das redes neurais envolvidas no processo ${ }^{7}$.

Em relação ao conteúdo, o cérebro memoriza as informações e as armazena por duas maneiras: sob a forma de memória declarativa (explícita) e memória não declarativa (implícita) ${ }^{8}$.

A memória implícita caracteriza-se por ser uma memória para habilidades motoras e perceptivas e outras formas de memória processual que envolve diferentes 
sistemas cerebrais: o cerebelo, o corpo estriado e a amígdala ${ }^{9}$. Ela é expressa principalmente em desempenho e não em palavras, sendo pouco modulada pelas emoções e estados de humor. Este tipo de memória relaciona-se com o lado não dominante do lobo temporal e é evocada principalmente para eventos que não são verbalizados. É uma memória rígida e se conecta fortemente às condições de estímulos originais, sob as quais a aprendizagem ocorreu ${ }^{10}$.

Nas memórias implícitas existe o priming. É uma memória que é evocada por meio de "dicas", isto é, alguns gestos, odores, sons, etc., podendo ser verbal ou perceptivo.

A existência do priming nas memórias implícitas demonstra que essas são adquiridas paralelamente de duas maneiras: uma envolve grandes conjuntos de estímulos e a outra utilizando fragmentos desse conjunto. A lembrança de palavras, objetos ou figuras melhora se houver exposição prévia a esses estímulos ${ }^{10}$.

O priming não necessita de nenhuma relação consciente de experiências prévias e compartilha algumas características com a memória de procedimento, onde atua aumentando as habilidades do indivíduo; e com a memória semântica, pois envolve representações cognitivas do ambiente, expressando-se mais cognitiva do que comportamentalmente ${ }^{11}$. É uma forma involuntária de memória que se dá através da identificação perceptiva de palavras e objetos ${ }^{12}$. 
O objetivo deste estudo foi avaliar a aquisição e persistência de memória implícita em adultos saudáveis, comparando o desempenho do teste Hooper Visual Organization Test (VOT) em dois grupos, com idades entre 30 e 40 anos e 60 a 70 anos.

\section{MÉTODO}

\section{Amostra}

Foi realizado um estudo transversal com indivíduos que frequentaram 0 ambulatório de terceira idade da unidade de neuropsicologia do hospital São Lucas da PUCRS, academias de ginástica e escolas de idiomas. A amostra foi composta de 100 indivíduos, 50 no grupo 1 (30 a 40 anos de idade) e 50 no grupo 2 (60 a 70 anos de idade). Para exclusão de utilização de medicação psiquiátrica e doença neurológica (doença demencial, AVC, tumor cerebral e malformações cerebrais), os sujeitos preencheram uma entrevista prévia. Este estudo foi aprovado pelo Comitê de Ética e Pesquisa em seres humanos da PUCRS, sob protocolo de pesquisa registro 10/05023. Após concordarem em participar, todos os indivíduos assinaram o Termo de Consentimento Livre e Esclarecido.

\section{Procedimento}

Para verificar o desempenho geral cognitivo, foi utilizado o Mini Exame do Estado Mental (MEEM), que é amplamente utilizado para triagem das funções cognitivas, 
fornecendo um parâmetro geral sobre as condições orientacionais, mnemônicas (memória de curto prazo), atencionais, práxicas, de linguagem e habilidades visuoespaciais $^{13}$. O ponto de corte utilizado para classificação de boa capacidade cognitiva foi de 24 pontos. Para classificar as classes sociais, foi utilizada a classificação socioeconômica $(A B E P)^{14}$, onde foram feitas atribuições de pesos a um conjunto de itens domésticos que proporcionam conforto como geladeira, televisor, rádio, videocassete/DVD, automóvel, empregado e da escolaridade do chefe da família. A soma dos pontos classificou os indivíduos nas classes sociais $A, B, C, D$ e $E$. Os sintomas de depressão foram avaliados através do inventário Beck de depressão $(B D I)^{15}$ e os sintomas de ansiedade foram avaliados pelo inventário Beck de ansiedade $(\mathrm{BAI})^{16}$, que se constituem de dois questionários de autorrelato. Os indivíduos responderam as questões e escolheram as afirmações e sintomas cognitivos e fisiológicos de ansiedade que mais se encaixavam com seu perfil. Para estimular a memória implícita foi administrado o Hooper Visual Organization Test (VOT) ${ }^{17}$ que é um instrumento utilizado para mensurar memória implícita através de estímulos compostos por fragmentos de figuras. É amplamente administrado em avaliação neuropsicológica. O VOT é um dos testes mais utilizados em pessoas idosas nas avaliações neuropsicológicas ${ }^{18}$.

Os estímulos foram apresentados em crescente grau de dificuldade e o indivíduo deve organizá-los mentalmente 
através da memória implícita e discriminação visual para que possa nomeá-los devidamente. O resultado foi 0 número total de acertos (1 ponto para cada resposta) e os índices foram obtidos através de um escore bruto que, após ser convertido em T-escore, classificaram o desempenho da memória implícita dos sujeitos em superior, médiosuperior, médio, médio-inferior e inferior.

\section{Análise Estatística}

A análise dos dados foi realizada utilizando o software SPSS versão 18.0. A idade e os escores de Hooper Visual Organization Test (VOT) foram descritos através de média e desvio padrão. As demais variáveis foram descritas através de frequências absolutas e relativas.

Para comparar as médias dos escores de VOT entre as faixas etárias, o teste t-Student foi utilizado. Para avaliar a associação entre as variáveis categóricas foi aplicado o teste qui-quadrado de Pearson ou exato de Fisher. Na complementação desses testes, a análise dos resíduos ajustados foi utilizada.

Para controlar fatores de confusão e avaliar fatores independentemente associados com a memória implícita de níveis médio inferior/inferior, a análise multivariada de Regressão de Poisson foi aplicada. A medida de associação de Razão de Prevalências (RP) juntamente com o intervalo de $95 \%$ de confiança foi utilizada para avaliar o efeito do fator em estudo. O nível de significância estatística considerado foi de $5 \%(p \leq 0,05)$. 


\section{RESULTADOS}

A amostra estudada foi composta por 100 indivíduos, sendo $50,0 \%$ com idades entre 30 e 40 anos e 50,0\% com idades entre 60 e 70 anos. A média de idade apresentada pelo grupo 1 (30-40) foi de $33,8 \pm 2,9$; enquanto que pelo grupo 2 (60-70) foi de $63,8 \pm 3,2$.

Quanto à escolaridade, os grupos não diferiram quanto à porcentagem de indivíduos: no grupo 2 prevaleceu 0 ensino superior $56,0 \%(n=56)$, seguido do ensino médio $44,0 \%(n=22)$, enquanto que no grupo $1,50,0 \%(n=50)$ tinham ensino superior e 50,0\% $(n=50)$ tinham ensino médio $(p=0,689)$.

Não houve diferença entre os grupos quanto aos sintomas ansiosos $(p=0,059)$. A proporção de indivíduos sem depressão e depressão leve também não foi entre os dois grupos: grupo 1- 94,0\% ( $n=47)$; grupo $2-96,0 \%$ $(n=48 ; p=0,557)$.

Os indivíduos do grupo 2 apresentaram maior T-escore no desempenho de VOT $(65,0 \pm 7,1$; entre 50 e 85), enquanto que o grupo 1 obteve menor T-escore $(57,1 \pm 6,1$; entre 47 e 75), demonstrando pior desempenho da memória implícita no grupo 2 de acordo com as médias de VOT $(p=<0,001)$.

Nas classificações qualitativas de VOT, o grupo 2 apresentou concentrações na classificação médio-inferior $34,0 \% \quad(n=17)$, enquanto que 0 grupo 1 apresentou classificações concentrando-se em superior $44,0 \%(n=22)$, 
demonstrando pior desempenho da memória implícita no grupo 2 nas classificações de VOT ( $p=<0,001$; Figura 1 ).

Figura 1. Comparação dos grupos em relação ao desempenho da memória implícita no Hooper Visual Organization Test (VOT).

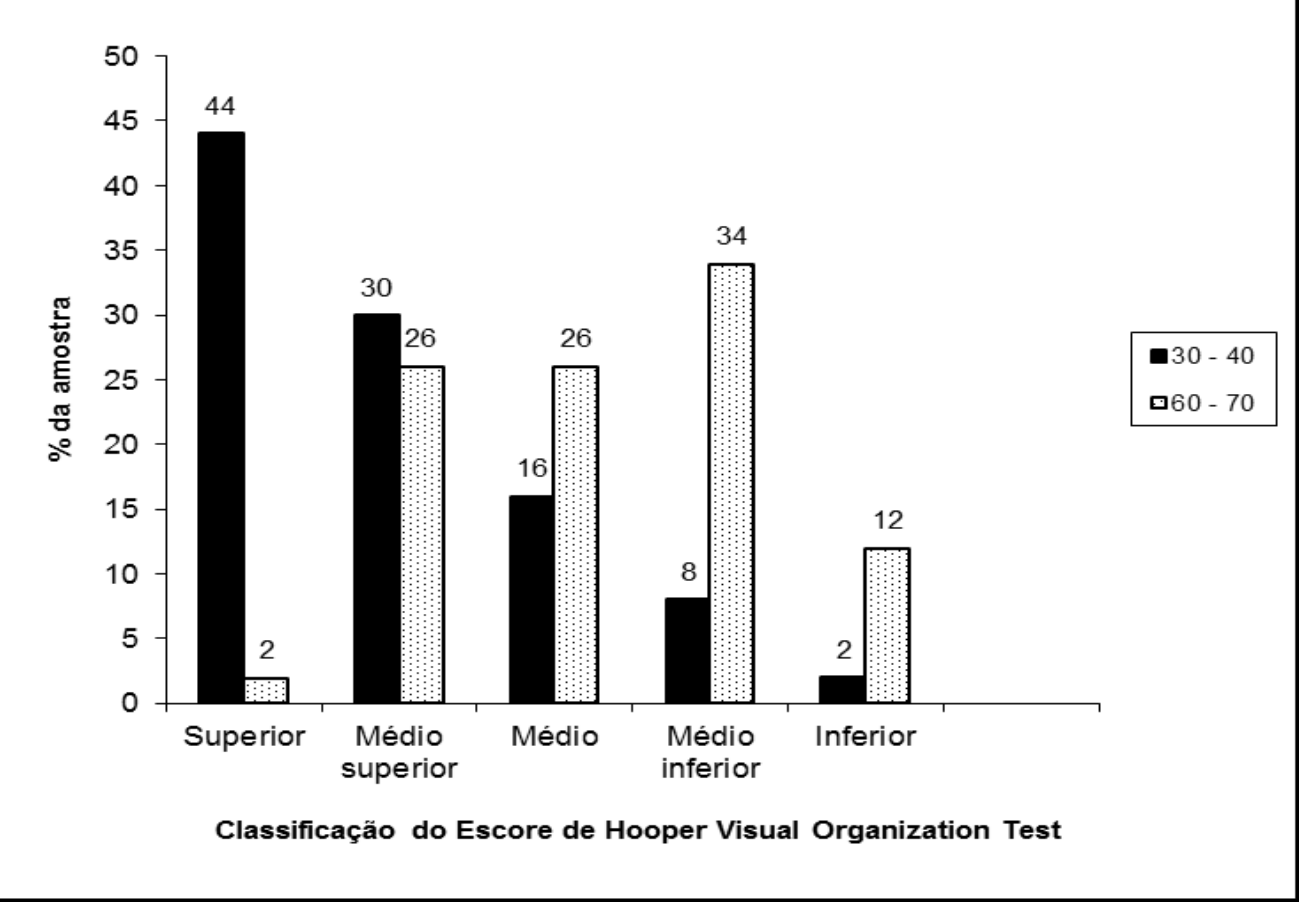

Em relação às faixas etárias, os resultados mostraram que o grupo 1 obteve maiores classificações qualitativas de VOT, concentrando-se em superior/médio-superior, com $72,5 \%(n=37)$, enquanto que o grupo 2 apresentou $27,5 \%$ $(n=14)$, para as mesmas classificações $(p=<0,001)$.

Quanto à classificação médio-inferior/inferior, o grupo 2 apresentou maiores escores, com $82,1 \% \quad(n=23)$, enquanto que 0 grupo 1 obteve $17,9 \% \quad(n=5)$, demonstrando que adultos mais velhos apresentaram pior desempenho em VOT ( $p=<0,001$; Tabela 1$)$. 
Tabela 1. Comparação entre as faixas etárias quanto ao gênero, escolaridade, classe socioeconômica, BAI, BDI e VOT.

\begin{tabular}{|c|c|c|c|}
\hline \multirow[t]{2}{*}{ Variáveis } & $\begin{array}{c}30 \text { a } 40 \text { anos } \\
(n=50)\end{array}$ & $\begin{array}{c}60 \text { a } 70 \text { anos } \\
(n=50)\end{array}$ & $\boldsymbol{P}$ \\
\hline & n (\%) & & \\
\hline $\begin{array}{cc}\text { Idade (anos) } & \text { Média } \pm \text { DP } \\
{[\min -\max ]}\end{array}$ & $\begin{array}{r}33,8 \pm 2,9 \\
{[30-40]}\end{array}$ & $\begin{array}{l}63,8 \pm 3,2 \\
{[60-70]}\end{array}$ & - \\
\hline \multicolumn{4}{|l|}{ Gênero } \\
\hline Masculino & $19(38,0)$ & $16(32,0)$ & $0,675 * *$ \\
\hline Feminino & $31(62,0)$ & $34(68,0)$ & \\
\hline \multicolumn{4}{|l|}{ Escolaridade } \\
\hline Médio & $25(50,0)$ & $22(44,0)$ & $0,689 * *$ \\
\hline Superior & $25(50,0)$ & $28(56,0)$ & \\
\hline \multicolumn{4}{|l|}{ ABEP } \\
\hline $\mathbf{A}$ & $14(28,0)$ & $10(20,0)$ & $0,624 * *$ \\
\hline B & $27(54,0)$ & $31(62,0)$ & \\
\hline C & $9(18,0)$ & $9(18,0)$ & \\
\hline \multicolumn{4}{|l|}{ BAI } \\
\hline Sem & $34(68,0)$ & $40(80,0)$ & $0,139 * *$ \\
\hline Leve & $9(18,0)$ & $9(18,0)$ & \\
\hline Moderada & $4(8,0)$ & $0(0,0)$ & \\
\hline Grave & $3(6,0)$ & $1(2,0)$ & \\
\hline \multicolumn{4}{|l|}{ BAI } \\
\hline Sem/Leve & $43(86,0)$ & $49(98,0)$ & $0,059 * * *$ \\
\hline Moderada/Grave & $7(14,0)$ & $1(2,0)$ & \\
\hline \multicolumn{4}{|l|}{ BDI } \\
\hline Sem & $41(82,0)$ & $41(82,0)$ & $0,557 * *$ \\
\hline Leve & $6(12,0)$ & $7(14,0)$ & \\
\hline Moderada & $3(6,0)$ & $1(2,0)$ & \\
\hline Grave & $0(0,0)$ & $1(2,0)$ & \\
\hline \multicolumn{4}{|l|}{ BDI } \\
\hline $\begin{array}{l}\text { Sem/Leve } \\
\text { Moderada/Grave }\end{array}$ & $47(94,0)$ & $\begin{array}{c}48(96,0) \\
2(4,0)\end{array}$ & $1,000 * * *$ \\
\hline $\begin{array}{l}\text { Moderada/Grave } \\
\text { Escore de VOT Média } \pm \text { DP } \\
\\
{[\text { min - max ] }}\end{array}$ & $\begin{array}{c}3(6,0) \\
57,1 \pm 6,1 \\
{[47-75]}\end{array}$ & $\begin{array}{l}2(4,0) \\
65,0 \pm 7,1 \\
{[50-85]}\end{array}$ & $<0,001^{*}$ \\
\hline Superior & $22(44,0)^{\#}$ & $1(2,0)$ & $<0,001 * *$ \\
\hline Médio superior & $15(30,0)$ & $13(26,0)$ & \\
\hline Médio & $8(16,0)$ & $13(26,0)$ & \\
\hline Médio inferior & $4(8,0)$ & $17(34,0)^{\#}$ & \\
\hline Inferior & $1(2,0)$ & $6(12,0)$ & \\
\hline
\end{tabular}

* Teste t-Student para amostras independentes;** Teste Qui-quadrado de Pearson;*** Teste exato de Fisher; ABEP: Classificação Socioeconômica; A: Classe socioeconômica; B: Classe socioeconômica; C: Classe socioeconômica; BAI: Inventário Beck de Ansiedade; BDI: Inventário Beck de Depressão; VOT: Hooper Visual Organization Test.

Com relação ao gênero, nos dois grupos, o desempenho de VOT não sofreu interferências $(p=0,473)$, bem como para escolaridade $(p=0,396)$ e classe socioeconômica $(p=0,191)$. 
Quanto aos sintomas psiquiátricos analisados nesse estudo, observou-se que não há associação com o desempenho da memória implícita para ansiedade $(p=0,363)$ ou depressão $(p=0,361)$.

Observou-se que os indivíduos do grupo 2 apresentam uma prevalência 4,64 vezes maior de classificações médio-inferior e inferior no VOT, quando comparados com o grupo 1 (Tabela 2).

Além disso, foi observado que a classe socioeconômica B apresenta uma prevalência 2,35 vezes maior nas classificações médio-inferior e inferior quando comparadas à classe $A$ e a classe $C$ apresenta uma prevalência 3,56 vezes maior para a mesma comparação. Porém, as classes socioeconômicas independem das variáveis quando ajustado o valor da razão de prevalência, pois os valores são praticamente os mesmos (Tabela 3 ).

\section{DISCUSSÃO}

Estudos sobre memória implícita e seu desempenho em humanos são de grande interesse para pesquisadores e clínicos, tendo em vista a dificuldade de se avaliar e mensurar esse tipo de memória com testes e tarefas neuropsicológicas.

Dentre as memórias de longa duração, a memória implícita é a memória que inclui habilidades motoras, sensitivas, intelectuais, associações, procedimentos e regras que são adquiridas através das experiências ${ }^{19,20}$ daí 
a importância dos estudos sobre o desempenho da memória implícita em humanos.

Tabela 2. Associação das variáveis em estudo com o desempenho da memória implícita.

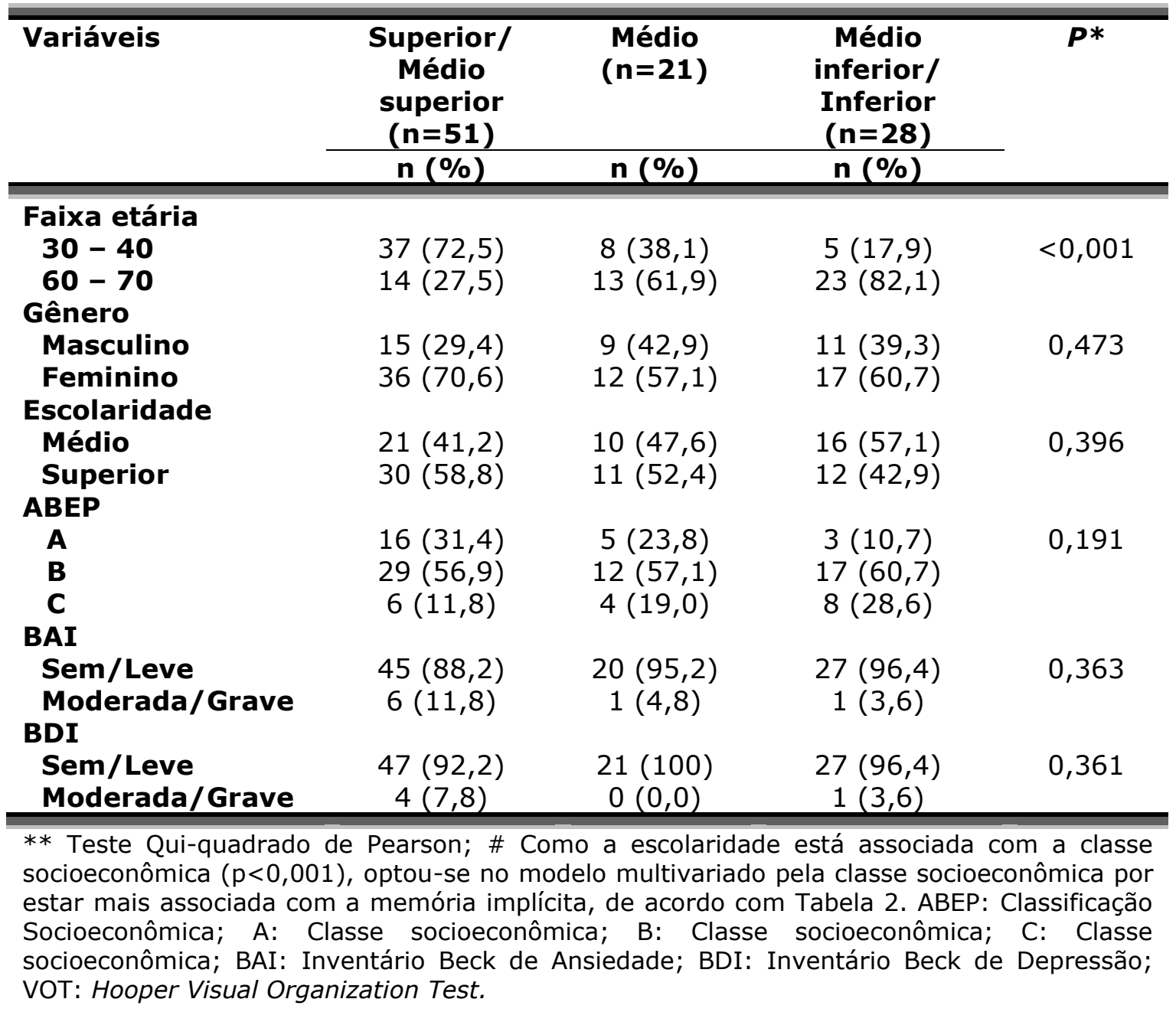

Tabela 3. Razão de Prevalências para desempenho de graus médio-inferior e inferior de memória implícita no teste VOT em relação à classe socioeconômica na análise univariada e pela regressão de Poisson.

\begin{tabular}{lcc}
\hline \hline Variáveis & $\mathrm{RP}_{\text {bruta }}(\mathrm{IC} 95 \%)$ & $\mathrm{RP}_{\text {aiustada** }}(\mathrm{IC} 95 \%)$ \\
\hline \hline Faixa etária 60 - 70 & $4,6(1,90-11,1)$ & $4,64(2,01-10,7)$ \\
Classe B* & $2,35(0,76-7,27)$ & $2,24(0,76-6,55)$ \\
Classe C* & $3,56(1,09-11,6)$ & $3,58(1,09-11,7)$ \\
\hline
\end{tabular}

* Em relação à classe socioeconômica $A$; ** ajustada para as variáveis BAI, BDI e gênero;

B: Classe socioeconômica; C: Classe socioeconômica. 
Nos últimos anos, muitos estudos apresentaram resultados controversos sobre o declínio dessa memória no envelhecimento ${ }^{21,22}$.

O pouco conhecimento sobre como avaliar o desempenho da memória implícita com testes e tarefas neuropsicológicas, motivou esse estudo, principalmente verificar se essa memória declina no envelhecimento, quando comparado a adultos jovens.

Seguindo os objetivos propostos no estudo, a seguir serão discutidos os resultados obtidos:

$\mathrm{Na}$ análise do escore ponderado, T-escore e classificações qualitativas de VOT em relação às faixas etárias, observou-se que o grupo 2 apresentou um pior desempenho da memória implícita, com T-escores altos e classificação concentrando-se em nível médio-inferior; enquanto que o grupo 1 apresentou T-escores baixos, ou seja, um melhor desempenho e classificação concentrandose em superior. Embora estudos discordem de que adultos mais velhos apresentam pior desempenho da memória implícita em comparação com adultos mais jovens $21,23,24$, nossos resultados mostraram ao contrário, isto é, através da análise das médias de idade, confirmamos a hipótese de que a partir dos 60 anos de idade a aquisição e persistência da memória implícita pode começar a apresentar declínios 22,25 .

Como utilizamos amostragem por conveniência, participaram do estudo sujeitos que frequentaram 0 ambulatório de terceira idade da unidade de 
neuropsicologia do HSL-PUCRS, academias de ginástica, escolas de idiomas e funcionários do HLS-PUCRS e a comparação dos grupos mostrou o predomínio da classe socioeconômica $B$, pois esses locais são frequentados por indivíduos de classes heterogêneas.

Sendo o BAI um instrumento adequado para avaliação de sintomas ansiosos, cabe aqui levantar questões sobre ansiedade, pois nosso estudo apresentou resultados interessantes sobre essa variável.

De acordo com estudos epidemiológicos, as mulheres com idades entre 25 a 44 anos apresentam maiores taxas de prevalência de transtornos de ansiedade do que os homens, sendo os transtornos psiquiátricos mais prevalentes na população ${ }^{26}$.

Em nosso estudo, não foi observado diferença entre os gêneros nos níveis de ansiedade e sim a diferença entre as faixas etárias, mostrando que o grupo 1 apresentou maior porcentagem de ansiedade moderada a grave, em comparação com o grupo 2, sugerindo que os adultos mais jovens parecem ter mais sintomas ansiosos do que adultos mais velhos nesta amostra.

As idades entre os grupos 1 e 2 foram fatores determinantes para melhor desempenho da memória implícita e pudemos observar que o grupo 1, o mais jovem, apresentou escores mais altos no VOT, mesmo com maiores índices de sintomas de ansiedade. Quanto à relação entre a interferência dos sintomas de ansiedade no 
desempenho da memória implícita, necessita-se maior comprovação dos dados para análise.

Fazendo uma correlação entre o desempenho da memória implícita e escolaridade, observamos que o grupo 2, com maior escolaridade (nível superior) mostrou pior desempenho no VOT. Entretanto, não houve diferença entre o desempenho no VOT e a variável escolaridade. Esses resultados já foram observados, porém em uma faixa etária mais jovem, entre 7 e 18 anos de idade, demonstrando que a escolaridade não influencia 0 desempenho da memória implícita mesmo nessa faixa etária ${ }^{27,28}$. Mesmo não apresentando o mesmo estímulo implícito, mas se tratando dos efeitos do priming em relação ao gênero, claros efeitos de priming de trechos de leitura foram observados para homens e mulheres, tanto para tarefa implícita afetiva quanto para tarefa implícita neutra ${ }^{29}$. Entretanto, o sexo masculino apresentou maior priming para conteúdos afetivos do que o sexo feminino. Em nosso estudo, observamos que o gênero parece não estar relacionado com o desempenho de indivíduos na memória implícita, pois não houve diferença entre os grupos.

A questão específica de comparação entre gênero, adultos e memória implícita não foi encontrada em pesquisas já desenvolvidas. O nosso estudo avaliou essa questão e para estudos futuros, propusemos essa nova contribuição. 
Como os estímulos do VOT são fragmentados e apresentam um crescente grau de dificuldade, os adultos mais velhos obtiveram pior desempenho no teste, com classificações qualitativas predominando em médioinferior/inferior $(82,1)$, enquanto que o grupo 1 apresentou somente $17,9 \%$ para as mesmas classificações. Esses resultados demostraram que a partir dos 60 anos de idade pode haver uma redução do priming, por ser uma memória evocada por meio de dicas e fazer parte da memória implícita ${ }^{5}$, mostrando que, mesmo com apresentação de pistas, não ocorre uma melhora no desempenho com o avanço da idade. Nossos resultados são corroborados com alguns estudos que referem à existência de uma redução do priming em idosos quando comparados a adultos mais jovens na realização de testes que exigem produção de uma resposta ${ }^{24}$.

De um modo geral, observamos que as classes socioeconômicas $A$, B e C não apresentaram relação com a memória implícita. Assim, podemos pensar que 0 desempenho dessa memória parece não sofrer influência direta da classe socioeconômica, pelo menos para essas classes, talvez por ser uma memória rígida e involuntária. Entretanto, quando comparamos as classes com o desempenho qualitativo médio-inferior e inferior de VOT das classes $B$ e $C$ em relação à classe $A$, os resultados mostraram aspectos relevantes os quais serão discutidos a seguir. 
O desempenho qualitativo de VOT apresentou diferenças em relação às classes socioeconômicas quando comparados os desempenhos mais baixos, isto é, nos graus médio-inferior e inferior.

$\mathrm{Na}$ comparação dos grupos, observamos que o grupo 2 apresentou maior prevalência de classificações médioinferior/inferior, quando comparados ao desempenho do grupo 1, bem como na comparação nas classificações socioeconômicas $A$ e $B$, que apresentaram maior prevalência para as mesmas classificações, quando comparadas à classe A. Porém, os sujeitos de classe C apresentaram a maior prevalência, quando comparada à classe A, sugerindo que a classe $C$, talvez por apresentar menores condições econômicas, seja mais privada de estímulos durante a vida, o que pode ter interferido no desempenho de VOT.

Podemos pensar, através dos resultados, que a classe socioeconômica A apresenta um melhor desempenho em VOT no que se refere às classificações qualitativas (médio, médio-superior e superior), quando comparadas classes $\mathrm{B}$ e C da amostra estudada, talvez por serem expostos a um ambiente mais rico de estímulos. Entretanto, o estudo não abrangeu todas as classes socioeconômicas, sendo necessária maior comprovação desses dados para uma análise mais detalhada. 


\section{CONCLUSÃO}

A memória implícita apresenta pior desempenho em adultos mais velhos quando comparados a adultos mais jovens, tanto para escores ponderados, como para classificação qualitativa de VOT, mostrando que a memória implícita apresenta declínio em idade avançada.

A escolaridade e o gênero não apresentaram influência no desempenho da memória implícita em adultos mais velhos.

Adultos mais jovens tiveram maior prevalência de ansiedade moderada a grave do que os adultos mais velhos, mas apresentaram melhor desempenho da memória implícita.

Não houve associação entre sintomas depressivos e desempenho da memória implícita em ambas as faixas etárias.

\section{AGRADECIMENTOS} recebido.

À CAPES, Ministério da Educação, pelo apoio financeiro

\section{REFERÊNCIAS}

1.Valle LELR, Capovilla, FC. Temas multidisciplinares de neuropsicologia e aprendizagem. São Paulo: Tecmedd, 2004,715p.

2.Squire LR, Kandel ER. Memória: da mente às moléculas. Porto Alegre: Artmed, 2003, 251p.

3.Yassuda MS, Lasca VB, Neri AL. Meta-memória e auto-eficácia: um estudo de validação de instrumentos de pesquisa sobre memória e envelhecimento. Psicol Reflex Crít 2005;18:78-90.

http://dx.doi.org/10.1590/S0102-79722005000100011

4.Izquierdo I. Memória. 2.ed. Porto Alegre: Artmed, 2011, 133p. 
5.Izquierdo I, Medina JH. Memory formation: the sequence of biochemical events in the hippocampus and its connection to activity in other brain structures. Neurobiol Learn Mem 1997;68:285-316. http://dx.doi.org/10.1006/nlme.1997.3799

6.Cammarota M, Bevilaqua LRM, Kerr D, Medina JH, Izquierdo I. Inhibition of mRNA and protein synthesis in the CA1 region of the dorsal hippocampus blocks reinstallment of a conditioned fear response. J Neurosci 2003;23:737-41. https://doi.org/10.1523/JNEUROSCI.23-03-00737.2003

7.Izquierdo I. Different molecular cascades in different sites of the brain control memory consolidation. Trends Neurosci 2006;29:496505. https://doi.org/10.1016/j.tins.2006.07.005

8.Squire LR, Zola-Morgan S. The medial temporal lobe memory system. Science, $1991 ; 253: 5026,1380-6$.

https://doi.org/10.1126/science.1896849

9.Kandel ER. The molecular biology of memory: CAMP, PKA, CRE, CREB-1, CREB-2, and CPEB. Mol Brain 2012;14:5-14. https://doi.org/10.1186/1756-6606-5-14

10.Kandel ER, Schwartz JH, Jessel TM. Princípios da neurociência. 4.ed. São Paulo: Manole, 2003, 1412p.

11.Shacter DL, Buckner RL. Priming and the brain. Neuron 1998;20:185-95. https://doi.org/10.1016/s0896-6273(00)80448-1

12.Tulving $E$, Schacter DL. Priming and human memory systems. Science 2002;247:301-6. https://doi.org/10.1126/science.2296719

13.Folstein MF, Folstein SE, McHugh PR. "Mini-Mental State": a practical method for grading the cognitive state of patients for the clinician. J Psychiatr Res 1975;12:189-98. https://doi.org/10.1016/0022-3956(75)90026-6

14.ABEP. Critério de Classificação Econômica Brasil, CCEB/2010 (endereço na internet). Brasil: Associação Brasileira de Empresas de Pesquisa (acessado em: 01/2011). Disponível em:

http://www.abep.org/novo/Content.aspx?ContentID =301

15.Beck AT, Steer RA, Garbin MG. Psychometric Properties of the Beck Depression Inventory: Twenty-Five Years of Evaluation. Clin Psychol Rev 1988;8:77-100. https://doi.org/10.1016/02727358(88)90050-5

16.Cunha JA. Manual da versão em português das Escalas Beck. São Paulo: Casa do Psicólogo, 2001, 170p.

17. Hooper HE. The Hooper visual organization test manual. In: Spreen O, Sherman MS, Strauss E. A compendium of neuropsychological tests: administration, norms and commentary. 3.rd. Oxford: University Press, 2006, p.508-12.

18.Greve KW, Lindberg RF, Bianchini KJ, Adams D. Construct validity and predictive value of the Hooper Visual Organization Test in stroke rehabilitation. Appl Neuropsychol 2000;7:215-22. https://doi.org/10.1207/S15324826AN0704 3

19.Anderson JR. Aprendizagem e memória: uma abordagem integrada. Rio de Janeiro: LTC, 2005, 327p. 
20.Purves D, Augustine GJ, Fitzpatrick D, Hall WC, LaMantia AS, McNamara JO, et al. Neurociências. 2.ed. Porto Alegre: Artmed, 2005, 476p.

21.Light LL. Memory and aging. In: Bjork EJ, Bjork RA, editors. Memory. San Diego: Academic Press; 1996, p.443-90.

22. La Voie DJ, Light LL. Adult age differences in repetition priming: a meta-analysis. Psychol Aging

http://dx.doi.org/10.1037/0882-7974.9.4.539

23. Light LL, La Voie D. Direct and indirect measures of memory in old age. In: Grafe P, Masson MEJ (Eds). Implicit memory: new directions in cognition, development, and neuropsychology. Hillsdale: Lawrence Erlbaum, 1993, p.207-30.

24.Davis HP, Bernstein PA. Age-related changes in explicit and implicit memory. In: Squire LR, Butters N (Eds). Neuropsychology of memory. (2.ed). New York: The Guilford Press, 1992, p.249-61.

25. Chiarello C, Hoyer WJ. Adult age differences in implicit and explicit memory: time courseand encoding effects. Psychol Aging 1988;3:358-66. http://dx.doi.org/10.1037//0882-7974.3.4.358

26. Lepine JP. The epidemiology of anxiety disorders: prevalence and societal costs. J Clin Psychiatry 2002;63(Suppl 14):4-8.

27.Tosello DJT. Contribuição para o estudo normativo do Hooper Visual Organization Test (VOT). Psicol Hosp 2005;3:59-83.

28. Lezak MD. The test-retest stability and reability of some tests commonly used in neuropsychological assessment. In: Spreen $\mathrm{O}$, Sherman MS, Strauss E. A compendium of neuropsychological tests: administration, norms, and commentary. 3.rd. Oxford: University Press, 2006, p.508-12.

29. Burton LA, Rabin L, Vardy SB, Frohlich J, Wyatt G, Dimitri D, et al. Gender differences in implicit and explicit memory for affective passages. Brain Cogn 2004;54:218-24.

http://dx.doi.org/10.1016/j.bandc.2004.02.011 